\title{
AURC All Normalized by Surface Area
}

National Cancer Institute

\section{Source}

National Cancer Institute. AURC All Normalized by Surface Area. NCI Thesaurus. Code C92344.

The area under the urinary excretion rate curve (AURC) from time zero to the last measurable rate divided by the surface area. 